\title{
Принципы конструирования магистрали DWDM на основе активной строительной длины оптического кабеля
}

\author{
К.Е. Заславский \\ Сибирский государственный университет телекоммуникаций и информатики, Новосибирск \\ *E-mail: zaslavskyke@mail.ru
}

DOI:10.31868/RFL2018.224-225

Волоконно-оптические магистрали с системами спектрального уплотнения (DWDM),содержащие усилители на OB с примесью эрбия (EDFA) широко применяются на сетях связи РФ. Однако, этим магистралям присущи следующие недостатки:

- так как пролёты длинные (более 100км), то и коэффициенты усиления усилителей велики, что приводит к значительным мощностям помех из-за спонтанного излучения,

- для достижения необходимой величины OSNR приходится выставлять значительный уровень передачи на выходе передатчика, что неизбежно увеличивает мощность нелинейных помех, возникающих в ОВ,

- с увеличением скорости передачи дистанция связи сокращается. В данной работе предлагается другой подход к конструированию магистрали, основанный на описании процесса распространения импульсов по ОВ уравнением Шредингера [1]:

$$
i d A / d z=-(i / 2) \alpha A+(1 / 2) \beta_{2}\left(d^{2} A / d t^{2}\right)-\gamma / A /^{2} A
$$

где А-медленно меняющаяся амплитуда огибающей электрического поля вдоль оси $\mathrm{z}, \beta_{2}$-дисперсия групповых скоростей, $\alpha$-потери, $\gamma$ - коэффициент нелинейности.

Уравнение (1) решается численными методами, например, Фурье-методом расщепления по физическим факторам (SSFM). При этом методе вся заданная дистанция связи разбивается на достаточно малые участки длиной $\mathrm{h}$, и на каждом участке рассчитывается изменение формы импульса, вызванное потерями, дисперсией и нелинейными явлениями в ОВ.

Предлагается рассматривать строительную длину ОК как физическую реализацию участка длиной h. Обычно она достаточно мала. Например, при дистанции связи $1=600 \kappa \mathrm{k}, \mathrm{h}=6 \kappa \mathrm{M}$. Для такой строительной длины уравнение (1) можно записать в виде:

$$
-(d A / d z)=(M+T+G+N(h))
$$

где М-оператор, характеризующий действие дисперсии ( $\beta 2)$, и потерь $(\alpha)$, T,Gоператоры, характеризующий действие компенсатора дисперсии и усилителя, соответственно, N(h) -оператор, характеризующий нелинейность строительной длины OB.

Таким образом, в отличие от уравнения (1). уравнение (2) предполагает, что в строительной длине осуществляется компенсация дисперсии и потерь. Назовём такую строительную длину активной (АСТ). 
Практически реализовать компенсатор дисперсии (КД) и оптический усилитель (ОУ), компенсирующий потери, можно в муфте, стыкующей АCТ. В качестве КД логично использовать волокно DCF (с отрицательной дисперсией), в качестве ОУ можно использовать либо EDFA, либо усилитель Рамана [2] (этот случай в данной работе не рассматривается). Предложена структурная схема соединений блоков в муфте, которая предусматривает разделение сигнала и накачки по длинам волн, независимые регулировки мощностей сигнала и дистанционной накачки, работу усилителя в линейном режиме, восстановление мощности сигнала на выходе муфты. Предложена конструкция магистрали, содержащая муфты оконечного усилителя, предусилителя и линейных усилителей. Для ввода-вывода сигнала и накачки в схемах передатчика и приёмника применяются стандартные мультиплексоры/демультиплексоры. Растёт OSNR показывает, что в предложенной конструкции нормативную вероятность ошибки в спектральном канале можно получить при более низких уровнях передачи сигнала, чем в существующих магистралях, что позволяет резко уменьшить влияние нелинейных помех на качество передачи информации.

\section{Литература}

[1] Г.П. Агравал, Нелинейная волоконная оптика. М.: Мир,324(1996)

[2] G.P. Agrawal, Application of Nonlinear Fiber Optics, Academic, 508 